'División de Enfermedades Cardiovasculares, Escuela de Medicina, Facultad de Medicina Pontificia Universidad Católica de Chile. Santiago, Chile. 2Unidad Docente Asociada de Ciencias de la Salud, Carrera de Nutrición, Facultad de Medicina, Pontificia Universidad Católica de Chile. Santiago, Chile. ${ }^{3}$ Departamento de Matemáticas, Universidad de la Serena. La Serena, Chile. aNutricionista, MSc en Fisiología Humana y Aplicada.

'Enfermera Matrona, MSc en Ciencias de la Salud. 'Enfermera Matrona. dEnfermera. eEstadístico, PhD.

Recibido el 29 de septiembre de 2016, aceptado el 14 de marzo de 2017.

Correspondencia a: Dra. Mónica Acevedo

División de Enfermedades Cardiovasculares, Escuela de Medicina, Facultad de Medicina, Pontificia Universidad Católica de Chile.

Portugal 61. Santiago, Chile. Teléfono: 562-23543334 macevedo@med.puc.cl

\section{Superioridad del nuevo puntaje de riesgo ACC/AHA 2013 por sobre el puntaje de Framingham, en la predicción de riesgo de mortalidad cardiovascular en Santiago}

\author{
MÓNICA ACEVEDO ${ }^{1}$, GIOVANNA VALENTINO ${ }^{1,2, a}$, \\ VERÓNICA KRAMER ${ }^{1, b}$, MARÍA JOSÉ BUSTAMANTE ${ }^{1, \mathrm{c}}$, \\ MARCELA ADASME ${ }^{1, \mathrm{~d}}$, LORENA ORELLANA ${ }^{\mathrm{l}, \mathrm{c}}$, \\ FERNANDO BARAONA ${ }^{1}$, CARLOS NAVARRETE ${ }^{3, e}$
}

\section{Evaluation the American College of Cardiology and American Heart Association Predictive score for cardiovascular diseases}

Background: Recently, the American College of Cardiology and American Heart Association (ACC/AHA) proposed a new cardiovascular risk (CV) score. Aim: To evaluate the new risk score (ACC/AHA 2013) in a Chilean population. Material and Methods: Between 2002 and 2014, body mass index (BMI), waist circumference, blood pressure, lipid profile and fasting blood glucose levels were measured and a survey about CV risk factors was responded by 3,284 subjects aged 40 to 79 years (38\% females), living in urban Santiago. ACC/AHA 2013, FRAM and Chilean FRAM scores were calculated. All-cause mortality was determined in July 2014 by consulting the Death Registry of the Chilean Identification Service, with an average follow up of $7 \pm 3$ years. Results: The prevalence of risk factors were $78 \%$ for dyslipidemia, 37\% for hypertension, 20\% for smoking, 7\% for diabetes, $20 \%$ for obesity and $54 \%$ for physical inactivity. The mean FRAM, Chilean FRAM and ACC/AHA scores were 8, 3 and 9\%, respectively. During follow-up, 94 participants died and 34 deaths were of cardiovascular cause. Participants who died had a higher prevalence of hypertension $(p<0.01)$ and diabetes $(p<0.01)$ and tended to be older $(p=0.06)$. The FRAM score for 10 years for deceased and surviving patients was 12 and $8 \%$, respectively ( $p=N S$ ). The figures for the Chilean FRAM were 5 and $2 \%$, respectively $(p=0.09)$. The figures for the ACC/AHA 2013 score were 33 and 9\%, respectively $(p=0.04)$. According to receiver operating characteristic curves, ACC/AHA 2013 had a higher area under de curve for CV mortality than FRAM and Chilean FRAM. Conclusions: The new ACC/AHA 2013 score, is better than traditional FRAM and Chilean FRAM scores in predicting cardiovascular mortality in a low risk population.

(Rev Med Chile 2017; 145: 292-298)

Key words: Preventive Medicine; Cardiovascular Diseases; Mortality; Risk Factors. 
L a enfermedad cardiovascular (CV) es la primera causa de muerte en el mundo ${ }^{1,2}$. Las cifras, sin embargo, en los países desarrollados han comenzado a disminuir, principalmente, por mejores programas de prevención CV primaria y secundaria. Por ello, los puntajes de predicción de riesgo se han convertido en herramientas importantes en la decisión de intervención, tanto sobre el estilo de vida, como farmacológico.

El puntaje global de Framingham (FRAM $)^{3}$ ha sido, y es, uno de los puntajes de riesgo más usados en la predicción de eventos CV en el mundo. Considera los principales factores de riesgo (FR) en sus ecuaciones y sólo incluye puntos finales duros coronarios a 10 años. La mayoría de las guías clínicas se basan en este puntaje con el fin de tratar los FR CV en forma oportuna. Sin embargo, se ha reportado que FRAM sobreestima el riesgo en poblaciones con bajas tasas de mortalidad CV, como la chilena. Por ello, en el año 2009, se adapta el puntaje FRAM a la población chilena, utilizando datos de la Encuesta Nacional de Salud (ENS) $2003^{4}$.

En el año 2013 fue publicado un nuevo puntaje, propuesto por el Colegio Americano de Cardiología (ACC) y la Asociación Americana del Corazón (AHA) ${ }^{5}$ llamado "New Pooled Cohort ASCVD Risk Equations". Este puntaje considera las mismas variables de FRAM y determina el riesgo a 10 años, pero agrega raza y propone como puntos finales duros, no sólo la enfermedad aterosclerótica coronaria, sino también la cerebrovascular, y sólo considera sujetos de 40 a 79 años para estimar el riesgo a 10 años. El riesgo alto es aquel $\geq 7,5 \%$ y define a aquellos sujetos que deberán tomar estatinas, a diferencia de FRAM, que es $\geq 20 \%$.

Este nuevo puntaje ACC/AHA ha sido muy criticado, debido a que clasifica una gran proporción de sujetos como "alto riesgo" y, por lo tanto, elegibles para el uso de estatinas, en contraste a FRAM ${ }^{6}$. Por otro lado, su validación ha sido escasa. Los pocos reportes que existen muestran que podría sobreestimar la ocurrencia de eventos ${ }^{7}$. De allí la necesidad de probar este nuevo puntaje en otras poblaciones.

En nuestro país no existen estudios al respecto. Por ello, el objetivo de este estudio fue determinar el poder de discriminación de mortalidad CV del puntaje ACC/AHA y compararlo con el puntaje de FRAM y el FRAM Chileno en una población chilena.

\section{Material y Métodos}

\section{Sujetos}

Estudio transversal en 3.284 sujetos mayores de 39 años (38\% mujeres, edad promedio $=57 \pm 10$ años), sin antecedente de enfermedad aterosclerótica demostrada, que consultan voluntariamente a un programa de cardiología preventiva entre los años 2002 y 2014.

\section{Recolección de datos}

A todos los sujetos se les interrogó sobre antecedentes demográficos, educacionales y médicos. Se midió: índice de masa corporal (IMC), perímetro de cintura y cadera, presión arterial sistólica y diastólica (PAS-PAD) según JNC VII, se tomó muestra de sangre venosa para medición de glicemia, perfil lipídico y proteína $\mathrm{C}$ reactiva ultrasensible (PCRus), y ergometría de esfuerzo máxima.

Se consideró hipertenso a todo sujeto con diagnóstico médico de hipertensión, con o sin tratamiento farmacológico $\mathrm{y}$, a aquellos con 2 o más determinaciones de PAS y $\mathrm{PAD} \geq 140 / 90$ en días alternos; dislipidémicos a aquellos con diagnóstico médico de hipercolesterolemia, con o sin tratamiento farmacológico, $\mathrm{y}$ a aquellos con colesterol total $\geq 200$ o HDL $<40 \mathrm{mg} / \mathrm{dL}$, en hombres $\mathrm{y}<50 \mathrm{mg} / \mathrm{dL}$ en mujeres, respectivamente; diabéticos a aquellos con diagnóstico médico de diabetes, con o sin tratamiento farmacológico y a los sujetos que fueran diagnosticados durante la evaluación. La capacidad aeróbica fue medida en equivalentes metabólicos por una ergometría de esfuerzo máxima, limitada por síntomas.

En todos los sujetos se calculó el nuevo puntaje de riesgo propuesto por ACC/AHA en $2013^{8}$. Este nuevo puntaje considera sexo, edad, raza (blanca no hispánica y afroamericana), colesterol total y HDL, PAS, tratamiento antihipertensivo, diabetes y tabaquismo, basándose en ecuaciones que estiman el riesgo CV a 10 años. Se considera alto riesgo un puntaje $\geq 7,5 \%$ a 10 años de un evento aterosclerótico, definido como: infarto al miocardio fatal o no fatal, accidente cerebrovascular ateroesclerótico fatal o no fatal, $u$ otra muerte cardiovascular. Además, se estimó el puntaje global de Framingham, según Wilson y cols ${ }^{3}$, calculando el riesgo de infarto o muerte por infarto a 10 años, que considera los mismos FR. Obviamente, utiliza otras ecuaciones para la estimación del riesgo 
y define alto riesgo a aquellos con $\geq 20 \%$ a 10 años. Difiere del otro puntaje, en cuanto a que no incluye eventos ateroscleróticos de los territorios no coronarios y no considera raza. Finalmente, se calculó el FRAM adaptado para la población chilena según Icaza y cols ${ }^{4}$.

La mortalidad cardiovascular y total fue determinada en julio de 2014 por consulta al Registro Civil, con un seguimiento promedio de $7 \pm 3$ años.

Todos los sujetos firmaron un consentimiento informado, aprobado por el Comité de Ética de la Facultad de Medicina de la Pontificia Universidad Católica de Chile.

\section{Mediciones de laboratorio}

Las muestras para perfil lipídico, glicemia y PCRus fueron obtenidas por punción venosa, con ayuno de $12 \mathrm{~h}$.

\section{Análisis estadístico}

Para las comparaciones de medias se ocupó análisis de varianza y modelos de regresión lineal múltiple. Los resultados se expresan como media o media \pm DE.
Se construyeron modelos de discriminación (habilidad de un marcador de diferenciar entre individuos que presentan o no el evento estudiado) en la predicción de riesgo de mortalidad cardiovascular, usando el nuevo puntaje ACC/ AHA 2013, el puntaje de FRAM y FRAM Chileno.

La capacidad discriminatoria de estos modelos fue evaluada a través de la construcción de curvas ROC (característica operativa del receptor) con sus áreas bajo las curvas correspondientes e intervalos de confianza de $95 \%$, usando mortalidad versus no mortalidad como punto final duro. Las curvas ROC están basadas en modelos de regresión logística. Los intervalos de confianza para el área bajo la curva (valor $\mathrm{C}$ ) fueron estimados mediante Bootstrap $^{8}$. Un valor $\mathrm{C}=0,50$ implica un valor predictivo igual al azar o "no discriminación".

Para todo el análisis estadístico se ocupó software R 2.14.

\section{Resultados}

En la Tabla 1 se muestran los datos demográficos y prevalencia de FR cardiovascular. Los

Tabla 1. Prevalencia de factores de riesgo cardiovascular tradicionales, niveles de proteína C-reactiva ultrasensible, capacidad aeróbica en METs, puntaje de riesgo ACC/AHA 2013, puntaje de Framingham chileno, puntaje global de Framingham y mortalidad total y por causa cardiovascular de los sujetos, separados por sexo

\begin{tabular}{|c|c|c|c|c|}
\hline & $\begin{array}{c}\text { Total } \\
(n=3.284)\end{array}$ & $\begin{array}{c}\text { Hombres } \\
(n=2.030)\end{array}$ & $\begin{array}{c}\text { Mujeres } \\
(n=1.254)\end{array}$ & $\mathbf{p}$ \\
\hline Edad (años) & $56,7 \pm 10$ & $55 \pm 10$ & $59 \pm 10$ & 0,06 \\
\hline Sedentarismo (\%) & 54 & 50 & 61 & $<0,0001$ \\
\hline Sobrepeso (\%) & 50 & 57 & 39 & $<0,0001$ \\
\hline Obesidad (\%) & 20 & 22 & 17 & $<0,001$ \\
\hline Diabetes mellitus (\%) & 7 & 8 & 6 & 0,02 \\
\hline Dislipidemia (\%) & 78 & 80 & 75 & $<0,01$ \\
\hline Tabaquismo (\%) & 20 & 19 & 21 & NS \\
\hline Hipertensión arterial (\%) & 37 & 37 & 37 & NS \\
\hline PCRus (mg/L) & $1,9 \pm 1,9$ & $1,9 \pm 1,8$ & $2 \pm 2$ & 0,09 \\
\hline PCRus > 2 mg/L (\%) & 32 & 30 & 36 & 0,05 \\
\hline METs máximos & $11 \pm 3$ & $13 \pm 3$ & $10 \pm 3$ & $<0,0001$ \\
\hline Puntaje AHA/ACC (\%) & $9 \pm 10$ & $10 \pm 10$ & $8 \pm 12$ & 0,09 \\
\hline Puntaje Framingham Chileno (\%) & $3,2 \pm 2$ & $2,6 \pm 2$ & $1,5 \pm 0,8$ & 0,02 \\
\hline Puntaje de Framingham (\%) & $8 \pm 7$ & $10 \pm 7$ & $5 \pm 4$ & 0,03 \\
\hline Mortalidad total $(n)$ & 94 & 62 & 32 & NS \\
\hline Mortalidad cardiovascular (n) & 34 & 22 & 12 & NS \\
\hline
\end{tabular}


sujetos eran de edad intermedia con alto porcentaje de sedentarismo, sobrepeso, obesidad y dislipidemia. El porcentaje de tabaquismo fue de $20 \%$, menor a la media nacional. Las mujeres presentaron significativamente menos obesidad, diabetes mellitus y dislipidemia, y más sedentarismo. La capacidad aeróbica máxima promedio de la muestra fue de $11 \pm 3$ METs, con una menor capacidad aeróbica en las mujeres versus hombres (10 versus 13 METs, $p<0,0001)$. La PCRus promedio fue de $1,9 \pm 1,9 \mathrm{mg} / \mathrm{L}(1,9 \pm 1,8 \mathrm{en}$ hombres y $2 \pm 2$ en mujeres, $\mathrm{p}=0,09$ ), con $32 \%$ de los sujetos con PCRus $\geq 2 \mathrm{mg} / \mathrm{L}$.

El nuevo puntaje ACC/AHA fue de $9 \pm 10 \%$ $(10 \pm 10 \%$ en hombres y $8 \pm 12 \%$ en mujeres, $\mathrm{p}=0,09)$. En contraste, el FRAM Chileno fue $3,2 \pm 2 \%(2,6 \pm 2 \%$ en hombres y $1,5 \pm 0,8 \%$ en mujeres, $\mathrm{p}=0,02)$ y el FRAM promedio fue de 8 $\pm 7 \%(10 \pm 7 \%$ en hombres y $5 \pm 4 \%$ en mujeres, $\mathrm{p}=0,03)$. Más de $50 \%$ de los hombres y mujeres presentaron un puntaje $\geq 7,5 \%$ según $\mathrm{ACC} / \mathrm{AHA}$, $y$, por lo tanto, tenían indicación de estatinas.

Durante el seguimiento promedio de $7 \pm 3$ años se reportaron 94 muertes por toda causa ( $\sin$ diferencias significativas por sexo), de las cuales, 34 fueron por causa CV (22 hombres y 12 mujeres) (Tabla 1). En la Tabla 2, se muestra que los sujetos fallecidos por causa cardiovascular eran de mayor edad y tenían mayor prevalencia de hipertensión arterial, diabetes mellitus y sedentarismo. Además, los fallecidos tuvieron significativamente menor capacidad aeróbica máxima que aquellos que permanecían vivos. Con respecto a los puntajes de riesgo, los fallecidos tenían significativamente mayor puntaje AHA/ACC (33\% versus $9 \%, \mathrm{p}=$ $0,04)$ y también mayor puntaje FRAM Chileno $(5 \%$ versus $2 \% ; \mathrm{p}=0,09)$ y FRAM $(12 \%$ versus $8 \%, \mathrm{p}=\mathrm{NS}$ ), aunque estas últimas diferencias no fueron estadísticamente significativas.

Finalmente, al evaluar la capacidad discriminatoria de los modelos de predicción de mortalidad $\mathrm{CV}$ construidos de manera independiente para 1) nuevo puntaje ACC/AHA 2013, 2) puntaje de FRAM y 3) puntaje FRAM Chileno, encontramos que el modelo para ACC/AHA fue superior en la predicción de mortalidad CV [C = 0,78 (95\% IC $0,68-0,84)$ versus $\mathrm{C}=0,60$ (95\% IC 0,52-0,74) versus $C=0,67$ (95\% IC 0,60-0,79), respectivamente], aunque no logró significancia estadística (Figura 1).

Tabla 2. Edad, prevalencia de factores de riesgo cardiovascular y valores promedio de proteína C-reactiva ultrasensible, capacidad aeróbica en METs, nuevo puntaje ACC/AHA 3013, puntaje de Framingham Chileno y puntaje global de Framingham en los vivos y fallecidos por causa cardiovascular

\begin{tabular}{|lccc|}
\hline & $\begin{array}{c}\text { Vivos } \\
\text { (n= 3.190) }\end{array}$ & $\begin{array}{c}\text { Fallecidos CV } \\
(\mathbf{n}=\mathbf{3 4 )}\end{array}$ & p \\
\hline Edad (años) & $56 \pm 10$ & $74 \pm 10$ & 0,06 \\
Sedentarismo (\%) & 54 & 70 & 0,08 \\
Obesidad (\%) & 20 & 24 & NS \\
Hipertensión arterial (\%) & 36 & 68 & $<, 001$ \\
Diabetes mellitus (\%) & 7 & 21 & $<, 01$ \\
Dislipidemia (\%) & 78 & 79 & NS \\
Tabaquismo (\%) & 20 & 18 & NS \\
Colesterol no HDL (mg/dl) & $155 \pm 40$ & $150 \pm 39$ & 0,08 \\
PCRus (mg/L) & $1,9 \pm 1,9$ & $2,8 \pm 2,3$ & $<0,0001$ \\
METs & $11 \pm 3$ & $8 \pm 3$ & 0,04 \\
\hline Puntaje AHA/ACC (\%) & $9 \pm 9$ & $33 \pm 19$ & 0,09 \\
\hline Framingham Chileno (\%) & $2 \pm 2$ & $5 \pm 3$ & NS \\
\hline Framingham (\%) & $8 \pm 6$ & $12 \pm 10$ & \\
\hline
\end{tabular}




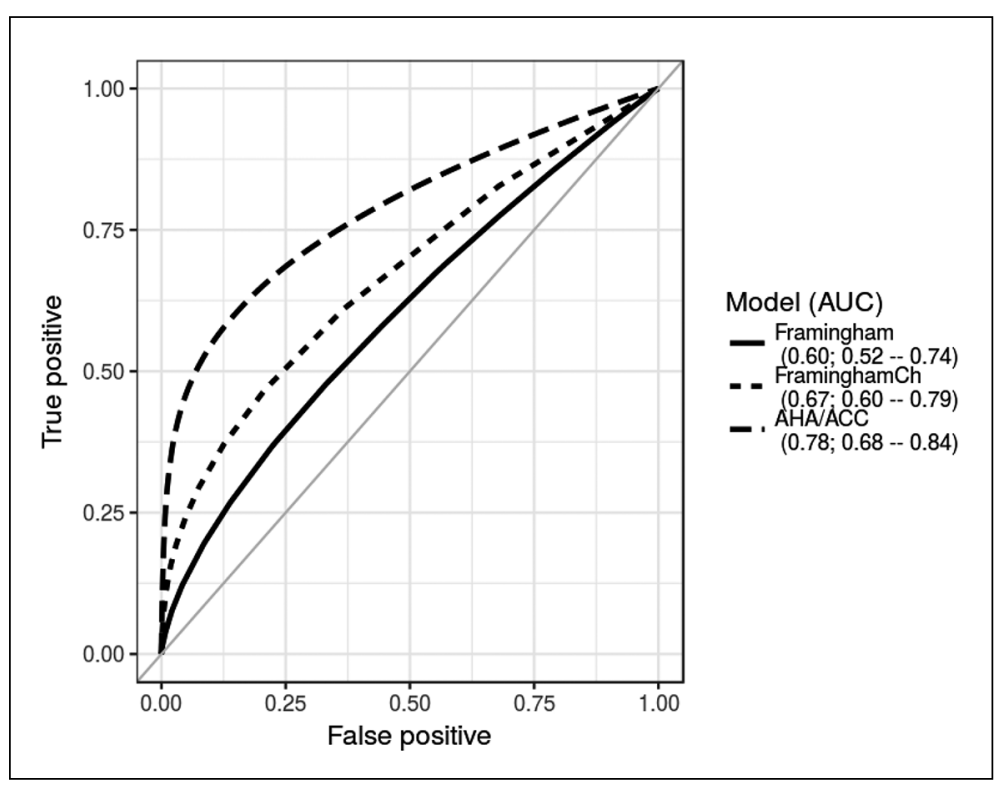

Figura 1. Curvas ROC para los modelos de predicción de riesgo de mortalidad: 1) puntaje global de Framingham; 2) puntaje de Framingham Chileno $y$ 3) nuevo puntaje ACC/AHA 3013.

\section{Discusión}

En este estudio demostramos superioridad del nuevo puntaje de estimación de riesgo cardiovascular ACC/AHA, por sobre el puntaje de FRAM y el FRAM Chileno en la discriminación de mortalidad CV. Los resultados sugieren que este nuevo puntaje podría ser de mayor utilidad en la práctica clínica convencional en la discriminación del riesgo de eventos CV en nuestra población, y constituyen el primer reporte de la aplicación de este nuevo puntaje en el país.

En noviembre de 2013, el ACC y la AHA publicaron las nuevas guías de manejo del colesterol para reducir eventos $\mathrm{CV}$, basados en el riesgo absoluto de cada individuo para la indicación de uso de estatinas ${ }^{5}$. Por ello, en forma concomitante, publicaron un nuevo puntaje de cálculo de riesgo basado en nuevas ecuaciones, reemplazando el antiguo puntaje de FRAM y el FRAM-ATP III. Este puntaje recibió el nombre de "New Pooled Cohort Equations", y calcula el riesgo de eventos CV duros a 10 años ${ }^{8}$. A diferencia de FRAM y FRAM Chileno, los eventos incluidos como puntos finales incluyen no sólo los eventos fatales y no fatales ateroescleróticos coronarios, sino, también, los cerebrovasculares. Asimismo, y también contrastando con FRAM, incluyó poblaciones de distintas áreas geográficas de Estados Unidos de Norteamérica y distintas razas ${ }^{10-14}$.

Este nuevo puntaje de riesgo AHA/ACC produjo mucha controversia, principalmente, por argumentarse que el poder de discriminación no era el óptimo, y que su calibración sobreestimaba el riesgo de eventos en comparación a lo observado $^{6}$. En este contexto, quisimos aplicar el nuevo puntaje en nuestra población. La población seleccionada fue una exenta de eventos vasculares (coronarios, periféricos o cerebrovasculares), del mundo real, o sea "no seleccionada" por muestreo epidemiológico, y que contaba con todas las variables usadas en el nuevo puntaje.

Los resultados de nuestro estudio (Tabla 1) muestran claramente que el puntaje de riesgo a 10 años, por FRAM, FRAM Chileno y AHA/ACC, es mayor en hombres. Sin embargo, el puntaje AHA/ACC muestra que la diferencia entre hombres y mujeres es menor que con FRAM y FRAM Chileno: el riesgo de la mujer en FRAM es casi la mitad que el de sus pares hombres (Tabla 1). Esto, sin duda, constituye una superioridad relativa del nuevo puntaje AHA/ACC en el subgrupo de mujeres, tradicionalmente subestimada en el riesgo de eventos en la mayoría de los puntajes existen- 
tes. Se deduce que, a pesar de que FRAM, FRAM Chileno y AHA/ACC usan las mismas variables, sus ecuaciones difieren en los valores, ya que el nuevo puntaje incluye poblaciones más diversas que el FRAM, que sólo incluyó la población de Framingham, la mayoría caucásicos.

En los sujetos fallecidos por causa CV de nuestro estudio, el puntaje AHA/ACC fue significativamente mayor que en los que permanecieron vivos, a diferencia de FRAM, que no resultó significativo (Tabla 2). En la Tabla 2 se ve que la edad sigue siendo el principal motor de riesgo, y a la vez, el más significativo de eventos $\mathrm{CV}$, lo que sucede con los puntajes AHA/ACC, FRAM y FRAM Chileno. Dentro de las otras variables, la única que influyó en la mortalidad CV fue la capacidad aeróbica.

La utilidad clínica de un puntaje de predicción de riesgo está determinada por su habilidad de discriminación y calibración ${ }^{15}$. En este estudio reportamos discriminación en mortalidad. Discriminación es la habilidad de un modelo de asignar mayor riesgo a los individuos que presentan el punto final o evento. En nuestro caso, mortalidad cardiovascular. Nosotros aquí no reportamos eventos vasculares ateroescleróticos no fatales, sino sólo mortalidad CV, ya que en nuestro país no existe un registro nacional obligatorio de eventos coronarios ni cerebrovasculares no fatales. Con respecto a los eventos fatales, $36 \%$ de las muertes fueron vasculares, existiendo muchos certificados de defunción con la causa de "paro cardiorrespiratorio", que no define etiología, pero que en las publicaciones internacionales se incorpora a la causa CV en su etiología. Dado que nuestra población es de bajo riesgo CV, y sólo hubo 94 fallecidos, al realizar un análisis utilizando sólo mortalidad total obtuvimos resultados similares, lo que concuerda con algunos autores que la definen como un punto final duro más potente que la muerte $\mathrm{CV}$, ya que su certeza es de $100 \%{ }^{16}$. La discriminación se realizó usando C-statistic. En general un C-index mayor a 0,7 es moderado a bueno en su capacidad discriminatoria. Claramente, como se observa en la figura, el C-index fue mejor para el nuevo puntaje AHA/ ACC en comparación a FRAM y FRAM Chileno. $\mathrm{Su}$ superioridad se evidencia en el valor absoluto del $\mathrm{C}=0,78$ para AHA/ACC versus 0,67 para FRAM Chileno y 0,60 para FRAM, sin embargo, al observar los intervalos de confianza, esta diferencia no fue significativa. El C-index para AHA/ACC de nuestro estudio fue similar al C-index reportado por Stone et $\mathrm{al}^{5} \mathrm{y}$ Goff et $\mathrm{al}^{8}$ en el trabajo en que se dieron a conocer las nuevas guías de colesterol y el nuevo puntaje de riesgo ACC/AHA. Es decir, en cuanto a mortalidad, AHA/ACC discriminó casi igual en la población americana que en la nuestra. En comparación a la literatura, el trabajo de Kavousi et $a{ }^{17} \mathrm{y}$ el de Muntner et a $\mathrm{l}^{18}$ son importantes de mencionar. Estos dos estudios tienen algunas ventajas sobre el nuestro: reportan eventos CV coronarios y cerebrovasculares, tanto fatales como no fatales. En el estudio de Muntner et $\mathrm{al}^{18}$, el C-index del nuevo puntaje AHA/ACC fue de 0,72. Ese trabajo concluye que esta nueva herramienta tiene un buen poder de discriminación. En el estudio de Kavousi ${ }^{17}$, que usó la población de Rotterdam, compararon el poder de discriminación y calibración de 3 puntajes: el AHA/ACC, el FRAM-ATP III, y el SCORE europeo. La discriminación de AHA/ ACC en esa población fue de $0,67(0,63-0,71)$ y el de FRAM-ATP III de 0,67 (0,62-0,72), pero el de SCORE europeo fue de $0,77(0,71-0,83)$. Es evidente que en nuestra población, FRAM fue mucho más débil en discriminar mortalidad CV que en la población de Rotterdam. Llama la atención, sin embargo, que el nuevo puntaje AHA/ACC presentara el mismo poder de discriminación que FRAM en los europeos. Esto es importante, ya que el nuevo puntaje de AHA/ACC no incluyó población hispánica, y pese a ello, nuestros resultados son muy semejantes a los de población americana.

Finalmente, en este estudio no realizamos calibración. Para ello es importantísimo en un futuro contar con un registro nacional de eventos $\mathrm{CV}$ confiable, y que debe ser obligatorio a nivel país. Este nos permitirá comparar los eventos predichos de los realmente observados.

Algunas limitaciones del estudio son: 1) bajo número de eventos fatales $\mathrm{CV}$, lo que no permitió análisis por sexo; 2) solo reportamos poder de discriminación de ACC/AHA y no de calibración; 3) población estudiada de estrato socioeconómico medio-medio alto, y, por lo tanto, no extrapolable a toda la Región Metropolitana.

\section{Referencias}

1. Go AS, Mozaffarian D, Roger VL, Benjamin EJ, Berry JD, Borden WB, et al. Executive summary: heart disease and stroke statistics-2013 update: a report from the American Heart Association. Circulation 2013; 127 (1): 143-52. 
2. Ministerio de Salud de Chile. Mortalidad por toda causa Chile 2011. http://www.deis.cl/?p=2541; 2011.

3. Wilson PW, D'Agostino RB, Levy D, Belanger AM, Silbershatz H, Kannel WB. Prediction of coronary heart disease using risk factor categories. Circulation 1998; 97 (18): 1837-47.

4. Icaza G, Núñez L, Marrugat J, Mujica V, Escobar MC, Jiménez AL, et al. Estimación de riesgo de enfermedad coronaria mediante la función de Framingham adaptada para la población chilena. Rev Med Chile 2009; 137 (10): 1273-82.

5. Stone NJ, Robinson JG, Lichtenstein $\mathrm{AH}$, Bairey $\mathrm{CN}$, Blum CB, Eckel RH, et al. 2013 ACC/AHA guideline on the treatment of blood cholesterol to reduce atherosclerotic cardiovascular risk in adults: a report of the American College of Cardiology/American Heart Association Task Force on Practice Guidelines. Circulation 2014; 129 (25 Suppl 2): S1-45.

6. Ridker PM, Cook NR. Statins: new American guidelines for prevention of cardiovascular disease. Lancet 2013; 382 (9907): 1762-5.

7. Karmali KN, Goff DC Jr, Ning H, Lloyd-Jones DM. A systematic examination of the 2013 ACC/AHA pooled cohort risk assessment tool for atherosclerotic cardiovascular disease. J Amer Coll Cardiol 2014; 64 (10): 959-68.

8. Goff DC Jr, Lloyd-Jones DM, Bennett G, Coady S, D'Agostino RB, Gibbons R, et al. 2013 ACC/AHA guideline on the assessment of cardiovascular risk: a report of the American College of Cardiology/American Heart Association Task Force on Practice Guidelines. Circulation 2014; 129 (25 Suppl 2): S49-73.

9. Carpenter J, Bithell J. Bootstrap confidence intervals: when, which, what? A practical guide for medical statisticians. Stats Med 2000; 19 (9): 1141-64.
10. The ARIC Investigators. The Atherosclerosis Risk in Communities (ARIC) Study: design and objectives. Am J Epidemiol 1989; 129 (4): 687-702.

11. Fried LP, Borhani NO, Enright P, Furberg CD, Gardin JM, Kronmal RA, et al. The Cardiovascular Health Study: design and rationale. Annals Epidemiol 1991; 1 (3): 263-76.

12. Friedman GD, Cutter GR, Donahue RP, Hughes GH, Hulley SB, Jacobs DR Jr, et al. CARDIA: study design, recruitment, and some characteristics of the examined subjects. J Clin Epidemiol 1988; 41 (11): 1105-16.

13. Dawber TR, Kannel WB, Lyell LP. An approach to longitudinal studies in a community: the Framingham Study. An New York Acad Sciences 1963; 107: 539-56.

14. Kannel WB, Feinleib M, McNamara PM, Garrison RJ, Castelli WP. An investigation of coronary heart disease in families. The Framingham offspring study. Am J Epidemiol 1979; 110 (3): 281-90.

15. Pencina MJ, D'Agostino RB. Overall $\mathrm{C}$ as a measure of discrimination in survival analysis: model specific population value and confidence interval estimation. Stats Med 2004; 23 (13): 2109-23.

16. Lauer MS, Blackstone EH, Young JB, Topol EJ. Cause of death in clinical research: time for a reassessment? J Amer Coll Cardiol 1999; 34 (3): 618-20.

17. Kavousi M, Leening MJ, Nanchen D, Greenland P, Graham IM, Steyeberg EW, et al. Comparison of application of the ACC/AHA guidelines, Adult Treatment Panel III guidelines, and European Society of Cardiology guidelines for cardiovascular disease prevention in a European cohort. JAMA 2014; 311 (14): 1416-23.

18. Muntner P, Colantonio LD, Cushman M, Goff DC, Howard GH, Horward VJ, et al. Validation of the atherosclerotic cardiovascular disease Pooled Cohort risk equations. JAMA 2014; 311 (14): 1406-15. 